Pacific

Journal of

Mathematics

\title{
A CHARACTERIZATION OF COMPLETELY 1-COMPLEMENTED SUBSPACES OF NONCOMMUTATIVE $L_{1}$-SPACES
}

Ping Wong Ng and Narutaka OzaWa 


\title{
A CHARACTERIZATION OF COMPLETELY 1-COMPLEMENTED SUBSPACES OF NONCOMMUTATIVE $L_{1}$-SPACES
}

\author{
Ping Wong Ng and Narutaka Ozawa
}

\begin{abstract}
A ternary ring of operators is an "off-diagonal corner" of a $C^{*}$-algebra and the predual of a ternary ring of operators (if it exists) is of the form $p R_{*} q$ for some von Neumann algebra $R$ and projections $p$ and $q$ in $R$. In this paper, we prove that a subspace of the predual of a ternary ring of operators is completely 1-complemented if and only if it is completely isometrically isomorphic to the predual of some ternary ring of operators. We next give an operator space characterization of the preduals of separable injective von Neumann algebras. Finally, we prove some concrete results about the finite dimensional completely 1-complemented subspaces of a von Neumann algebra predual.
\end{abstract}

\section{Introduction.}

Douglas proved that the 1-complemented subspaces of $L_{1}(X)$ are just the subspaces which are isometric to $L_{1}(Y)$ for some measure space $Y$ (see [D]). In this paper we show that there is a natural "noncommutative" analogue of this result. In order to state the general result, it is necessary to consider the preduals of spaces of the form $q R p$ where $q$ and $p$ are projections in a von Neumann algebra $R$. These spaces are closed under the trilinear operation $(x, y, z) \rightarrow x y^{*} z$, and they are known as ternary rings of operators or simply TROs (see $[\mathbf{E O R}],[\mathbf{H}]$ and $[\mathbf{K} \mathbf{1}]$ ).

Moreover, in order to generalize Douglas' result, we need to consider all the spaces above as operator spaces. An operator space is a linear space $X$ of operators on some Hilbert space $\mathcal{H}$, which is equipped with distinguished matrix norms from $\mathbb{B}(\mathcal{H})$. More precisely, each matrix space $\mathbb{M}_{n}(X)$ has a canonical norm determined by the operator norm on

$$
\mathbb{M}_{n}(\mathbb{B}(\mathcal{H})) \cong \mathbb{B}\left(\mathcal{H}^{n}\right) .
$$

This concrete definition of operator spaces was given by Arveson (see [Arv]). The morphisms between operator spaces are completely bounded maps. A linear map of operator spaces $\phi: X \rightarrow Y$ determines linear mappings

$$
\phi_{n}: \mathbb{M}_{n}(X) \rightarrow \mathbb{M}_{n}(Y):\left[x_{i j}\right] \rightarrow\left[\phi\left(x_{i j}\right)\right]
$$


and $\phi$ is completely bounded if

$$
\|\phi\|_{c b}={ }_{\mathrm{df}} \sup \left\{\left\|\phi_{n}\right\|: n \geq 1\right\}<\infty .
$$

Ruan abstractly characterized operator spaces in terms of their matrix norms (see $[\mathbf{R}]$ ). Operator spaces are the natural noncommutative quantizations of Banach spaces. There are many parallel results as well as many distinctions between operator spaces and Banach spaces. Nevertheless, Banach spaces provide an important source of inspiration for the development of operator spaces.

Given an inclusion $X \subseteq Y$ of operator spaces, we say that $X$ is completely 1-complemented in $Y$, if there is a completely contractive projection on $Y$ whose range is $X$. In $\S 3$ we prove the following noncommutative generalization of Douglas' result that the 1-complemented subspaces of an $L_{1}$ space are exactly those subspaces which are isometric to $L_{1}$ spaces:

Theorem 1.1. Let $X$ be a subspace of the predual of a TRO. Then, $X$ is completely 1-complemented if and only if $X$ is completely isometric to the predual of some TRO.

We note that various analogous results have been proven for subspaces of a von Neumann algebra predual which are 1-complemented (the projection is only contractive; see $[\mathbf{A F 1}],[\mathbf{A F 2}],[\mathbf{A F} 3]$ and $[\mathbf{K 2}])$. This theory is more complicated and less definitive than that considered in the above theorem.

As a consequence of this result, we have:

Corollary 1.2. Let $R_{1}$ be an injective von Neumann algebra. Let $R_{2}$ be an arbitrary von Neumann algebra. Suppose that $\left(R_{2}\right)_{*}$ can be completely isometrically embedded into $\left(R_{1}\right)_{*}$. Then $R_{2}$ must be injective.

In $\S 4$, we give an operator space characterization of the preduals of separable injective von Neumann algebras. This extends the work of [ER2], which is in turn a generalization of the following classical result, which gives a characterization of the $L_{1}$ spaces (see [ER2] for definitions and notation):

Theorem 1.3. Let $X$ be a Banach space. Then $X$ is isometric to an $L_{1}$ space if and only if $X$ is $\mathcal{L}_{1,1+}$.

To state our theorem, we need to first give some preliminary definitions.

Definition 1.4. We say that an operator space $X$ is a rigid $\mathcal{O} \mathcal{L}_{1,1+}$ space if given elements $\omega_{1}, \ldots, \omega_{s} \in X$ and $\epsilon>0$, we can find positive integers $n_{i}$ and a complete isometry

$$
S: \mathbb{T}_{n_{1}} \oplus \cdots \oplus \mathbb{T}_{n_{k}} \rightarrow X
$$

with

$$
\operatorname{dist}\left(\omega_{j}, S\left(\mathbb{T}_{n_{1}} \oplus \cdots \oplus \mathbb{T}_{n_{k}}\right)\right)<\epsilon
$$

for all $j$. 
Definition 1.5. Let us suppose that $\lambda>1$. We say that an operator space $X$ is an $\mathcal{O} \mathcal{L}_{1, \lambda}$ space if for every finite dimensional subspace $E$ of $X$, there exists a finite dimensional subspace $F$ with $E \subseteq F \subseteq X$ such that

$$
d_{c b}\left(F, \mathbb{T}_{n_{1}} \oplus \cdots \oplus \mathbb{T}_{n_{k}}\right)<\lambda
$$

for some integers $n_{i}$. We are using Pisier's operator space analogue of the Banach-Mazur distance:

$$
d_{c b}(X, Y)=_{\mathrm{df}} \inf \left\{\|T\|_{c b}\left\|T^{-1}\right\|_{c b}: T: X \rightarrow Y \text { a linear homeomorphism }\right\},
$$

where \|\|$_{c b}$ is the completely bounded norm.

Definition 1.6. We say that an operator space $X$ is an $\mathcal{O} \mathcal{L}_{1,1+}$ space if it is $\mathcal{O} \mathcal{L}_{1, \lambda}$ for every $\lambda>1$.

What we prove is the following:

Theorem 1.7. Let $X$ be a separable operator space. Then the following are equivalent:

(1) $X$ is completely isometric to the predual of an injective von Neumann algebra.

(2) $X$ is rigid $\mathcal{O} \mathcal{L}_{1,1+}$.

(3) $X$ is $\mathcal{O} \mathcal{L}_{1,1+}$.

In Theorem 1.7, the condition of separability is necessary, for if $\mathcal{H}$ has countably infinite dimension, and $\mathcal{K}$ has uncountable dimension, as Hilbert spaces, then $\mathbb{T}(\mathcal{H}, \mathcal{K})$ is an example of an $\mathcal{O} \mathcal{L}_{1,1+}$ space which is not a von Neumann algebra predual,

Though the proof of Theorem 1.7 is short, it relies on [ER2], and a deep result from $[\mathbf{E O R}]$. We leave open the question of whether the preduals of dual injective operator spaces (which are automatically TROs) have the rectangular $\mathcal{O} \mathcal{L}_{1,1+}$ property.

In $\S 5$, we investigate concrete procedures for finding completely 1-complemented subspaces of the predual $R_{*}$ of a von Neumann algebra $R$. A representative example of this is the following:

Proposition 1.8. Let $S: \mathbb{T}_{n, m} \rightarrow R_{*}$ be a complete isometry. Then there exists partial isometries $\left\{u_{j}: 1 \leq j \leq m\right\}$ and $\left\{w_{i}: 1 \leq i \leq n\right\}$ such that:

(1) $r\left(u_{j}\right)=r\left(S\left(e_{1,1}\right)\right)$ and $r\left(w_{i}\right)=l\left(S\left(e_{1,1}\right)\right)$ for all $i, j$,

(2) the projections $\left\{l\left(u_{j}\right)\right\}$ are pairwise orthogonal,

(3) the projections $\left\{l\left(w_{i}\right)\right\}$ are pairwise orthogonal,

(4) $S\left(e_{i, j}\right)=w_{i} S\left(e_{1,1}\right)\left(u_{j}\right)^{*}$, for all $i, j$.

In the statements above, for an element $x \in R, l(x)$ and $r(x)$ are the left and right support projections of $x$ respectively. Similarly, for $\omega \in R_{*}, l(\omega)$ and $r(\omega)$ are the left and right support projections of $\omega$ respectively. 
A result of this type completely characterizes the finite dimensional spaces in question. (See Propositions 5.1 and 5.2.)

Using these calculations, one can construct specific examples of the projections in Theorem 1.1. In particular, in the context of Proposition 1.8, the formula

$$
p(\omega)={ }_{\mathrm{df}} \sum_{i, j}\left\langle\omega \mid v\left(S\left(e_{i, j}\right)\right)\right\rangle S\left(e_{i, j}\right)
$$

gives a completely contractive projection from $R_{*}$ onto $S\left[\mathbb{T}_{n, m}\right]$ (here $v\left(S\left(e_{i, j}\right)\right)$ is the partial isometry gotten from the polar decomposition of the normal linear functional $\left.S\left(e_{i, j}\right)\right)$. We note that the dual map $\left(S^{-1} \circ p\right)^{*}$ : $\mathbb{M}_{n, m} \rightarrow R$ is the TRO-homomorphism given by mapping $e_{i, j} \mapsto v\left(S\left(e_{j, i}\right)\right)^{*}$. The projection $p$ above generalizes the conditional expectations in the classical $L_{1}$ theory. Furthermore, we show that the completely contractive projections on $R_{*}$, with finite dimensional range, are exactly the perturbations of these "noncommutative conditional expectations" (see Theorem 6.4 and Theorem 6.5). This is a partial generalization of a result of Douglas (see $[\mathbf{D}])$. As an example of this, we have:

Proposition 1.9. Suppose that $p$ is a completely contractive projection on $R_{*}$, with finite dimensional range $X \subseteq R_{*}$. Then

$$
p=q+T,
$$

where $q$ is a projection of the type similar to that of $(*)$, and $\|T\|_{c b} \leq 1, T^{2}=$ $0, T=p T=q T=T F(X)$. The completely contractive projection $F(X)$ : $R_{*} \rightarrow R_{*}$ is defined in the second last paragraph of Section 2 (Definitions and notation). Intuitively, $F(X)$ is the natural projection onto the subspace of $R_{*}$ which is orthogonal to $X$.

Conversely, any map $R_{*} \rightarrow R_{*}$ of the type stated in Proposition 1.9 is a completely contractive projection on $R_{*}$, with finite dimensional range (see Theorem 6.5). We note that in Proposition 1.9, the "perturbation" $T$ is actually given concretely by $T={ }_{\mathrm{df}} p F(X)$. Hence, $T$ is the part of the projection $p$ which maps the subspace (of $R_{*}$ ) which is orthogonal to $X$ into $X$.

\section{Definitions and notation.}

In this section, we establish some definitions and the notation that will be used in the paper (for details, see $[\mathbf{R}]$ and $[$ Tak $]$ ).

An operator space $V$ is a linear space of bounded linear operators on a Hilbert space $H$. Each matrix space $\mathbb{M}_{n}(V)$ has a canonical norm determined by the operator norm on

$$
\mathbb{M}_{n}(\mathbb{B}(\mathcal{H}))=\mathbb{B}\left(\mathcal{H}^{n}\right) .
$$


These matrix norms are a central distinguishing feature in operator space theory, and they are used in Ruan's axiomatization of operator spaces (see $[\mathbf{R}])$.

A linear map $\phi: V \rightarrow W$ between operator spaces determines linear mappings

$$
\phi_{n}: \mathbb{M}_{n}(V) \rightarrow \mathbb{M}_{n}(W):\left[v_{i, j}\right] \mapsto\left[\phi\left(v_{i, j}\right)\right] .
$$

This, together with the matrix norms (defined in the previous paragraph) gives the completely bounded norm of $\phi$ :

$$
\|\phi\|_{c b}={ }_{\mathrm{df}} \sup \left\{\left\|\phi_{n}\right\|: n \geq 1\right\} .
$$

With the above, we now say that:

(1) $\phi$ is completely bounded if $\|\phi\|_{c b}<\infty$,

(2) $\phi$ is completely contractive if $\|\phi\|_{c b} \leq 1$, and

(3) $\phi$ is completely isometric if $\phi_{n}$ is isometric for all $\mathrm{n}$.

Finally, for operator spaces $V$ and $W$, we will let " $C B(V, W)$ " denote the set of completely bounded maps from $V$ to $W$. We note that $C B(V, W)$ is an operator space, with matrix norms given by the identifications

$$
\mathbb{M}_{n}(C B(V, W))=C B\left(V, \mathbb{M}_{n}(W)\right) .
$$

Let $R$ be a von Neumann algebra.

(1) $R^{*}$ is an operator space with matrix norms given by the identifications

$$
\mathbb{M}_{n}\left(R^{*}\right)=C B\left(R, \mathbb{M}_{n}(\mathbb{C})\right) .
$$

(2) Let $R_{*}$ will be the unique predual of $R$. $R_{*}$ is an operator space, with operator norms inherited from $R^{*} \supseteq R_{*}$.

(3) For an element $x \in R,\|x\|_{\infty}$ will be its $C^{*}$-algebra norm. The subscript will be dropped whenever the context is clear.

(4) $l(x)$ and $r(x)$ will be the left support and right support projections, respectively, of $x$.

(5) $v(x)$ and $|x|$ will be the partial isometry and the positive element of $R$, respectively, obtained from the polar decomposition of $x$.

$\mathbb{T}_{n_{1}, m_{1}}(\mathbb{C}) \oplus \cdots \oplus \mathbb{T}_{n_{1}, m_{1}}(\mathbb{C})$ will be the operator space dual of the direct sum of rectangular matrices $\mathbb{M}_{n_{1}, m_{1}}(\mathbb{C}) \oplus \cdots \oplus \mathbb{M}_{n_{k}, m_{k}}(\mathbb{C})$ (and hence, $\mathbb{T}_{n_{1}, m_{1}}(\mathbb{C}) \oplus \cdots \oplus \mathbb{T}_{n_{k}, m_{k}}(\mathbb{C})$ is an operator space whose Banach space level norm is the $l^{1}$ direct sum of trace class norms). Throughout this paper, we will drop the "CC"s and hence write "T $\mathbb{T}_{n_{1}, m_{1}} \oplus \ldots \mathbb{T}_{n_{k}, m_{k}}$ " and "M $\mathbb{M}_{n_{1}, m_{1}} \oplus \cdots \oplus \mathbb{M}_{n_{k}, m_{k}}$ ".

$\left\{e_{i, j}: 1 \leq i \leq n, 1 \leq j \leq m\right\}$ will be the matrix units of $\mathbb{T}_{n, m}\left(=\mathbb{M}_{n, m}\right.$ setwise), where $e_{i, j}$ is 1 in the $(i, j)$-th position and zero everywhere else. More generally, $\left\{e_{i, j}^{l}: 1 \leq i \leq n_{l}, 1 \leq j \leq m_{l}, 1 \leq l \leq k\right\}$ will be the matrix units of $\mathbb{T}_{n_{1}, m_{1}} \oplus \ldots \oplus \mathbb{T}_{n_{k}, m_{k}}\left(=\mathbb{M}_{n_{1}, m_{1}} \oplus \ldots \oplus \mathbb{M}_{n_{k}, m_{k}}\right.$ setwise), where $e_{i, j}^{l}$ is 1 in the $(i, j)$-th position of the $\mathbb{T}_{n_{l}, m_{l}}$ summand and zero everywhere else. 
Let $\omega$ and $\gamma$ be normal linear functionals in $R_{*}$.

(1) If $\omega$ is self-adjoint, $\operatorname{supp}(\omega)$ is its support projection.

(2) $l(\omega)$ and $r(\omega)$ are the left support and right support projections, respectively, of $\omega$.

(3) $\omega \perp \gamma$ will mean that $l(\omega) \perp l(\gamma)$ and $r(\omega) \perp r(\gamma)$.

(4) $\|\omega\|_{1}$ will be the norm of $\omega$, in $R_{*}$. The subscript will be dropped whenever the context is clear.

(5) $v(\omega)$ and $|\omega|$ will be the partial isometry in $R$ and the positive element in $R_{*}$, respectively, obtained from the polar decomposition of $\omega$.

(6) For an element $x$ of $R,\langle\omega \mid x\rangle$ will be $\omega\left(x^{*}\right)$.

For a finite dimensional subspace $X$ of $R_{*}$, let

$$
l(X)={ }_{\mathrm{df}} \sup \{l(\omega): \omega \in X\},
$$

and let

$$
r(X)={ }_{\mathrm{df}} \sup \{r(\omega): \omega \in X\} .
$$

Let $E(X)$ be the completely contractive projection on $R_{*}$ given by $E(X)(\gamma)$ $={ }_{\mathrm{df}} l(X) \gamma r(X)$ for all $\gamma \in R_{*}$ Let $F(X)$ be the completely contractive projection on $R_{*}$ given by $F(X)(\gamma)=_{\text {df }}(1-l(X)) \gamma(1-r(X))$ for all $\gamma \in R_{*}$.

In this paper, $V$ and $W$ will always be ternary rings of operators. "SOT" and "WOT" will always denote the strong and weak operator topologies, respectively, on some Hilbert space.

\section{Main theorem.}

A ternary ring of operators (TRO) is a closed subspace of $\mathbb{B}(\mathcal{K}, \mathcal{H})$ which is closed under the ternary product

$$
(x, y, z) \mapsto x y^{*} z .
$$

We say that a map between TROs is a TRO-homomorphism (resp. TROisomorphism) if it preserves the ternary product (resp. and moreover is injective). We note that a TRO-homomorphism (resp. TRO-isomorphism) is automatically completely contractive (resp. completely isometric). See $[\mathbf{H}]$, $[\mathbf{K 1}]$ and $[\mathbf{E O R}]$ for systematic studies of TROs.

Let $V^{\sharp}={ }_{\text {df }}\left\{x \in \mathbb{B}(\mathcal{H}, \mathcal{K}): x^{*} \in V\right\}$; and let $V \subseteq \mathbb{B}(\mathcal{K}, \mathcal{H})$ be a nondegenerate TRO, i.e., $\overline{V \mathcal{K}}=\mathcal{H}$ and $\overline{V^{\sharp} \mathcal{H}}=\mathcal{K}$. Then, $V V^{\sharp}$ and $V^{\sharp} V$ are nondegenerate $*$-subalgebras of $\mathbb{B}(\mathcal{H})$ and of $\mathbb{B}(\mathcal{K})$ respectively. We define the $C^{*}$-algebra $C$ in $\mathbb{B}(\mathcal{H} \oplus \mathcal{K})$ by

$$
C={ }_{\mathrm{df}}\left[\begin{array}{cc}
\overline{V V^{\sharp}+\mathbb{C} 1} & \frac{V}{V^{\sharp}} \\
V^{\sharp} V+\mathbb{C} 1
\end{array}\right]
$$


and projections $p_{\mathcal{H}}$ and $p_{\mathcal{K}}$ in $C$ by

$$
p_{\mathcal{H}}={ }_{\mathrm{df}}\left[\begin{array}{ll}
1 & 0 \\
0 & 0
\end{array}\right] \text { and } p_{\mathcal{K}}={ }_{\mathrm{df}}\left[\begin{array}{ll}
0 & 0 \\
0 & 1
\end{array}\right] .
$$

Then, $V$ is TRO-isomorphic to the corner $p_{\mathcal{H}} C p_{\mathcal{K}}$ of the $C^{*}$-algebra $C$. We note that $C$ is finite dimensional if $V$ is a finite dimensional TRO.

In [EOR], Effros, Ozawa and Ruan proved that a TRO which is a dual space as a Banach space has a unique predual and is weak*-homeomorphically TRO-isomorphic to $p R q$ for some von Neumann algebra $R$ and projections $p$ and $q$ in $R$. Thus, the predual of a dual TRO is of the form $p R_{*} q$.

Theorem 1.1. Let $X$ be a subspace of the predual of a TRO. Then, $X$ is completely 1-complemented if and only if $X$ is completely isometrically isomorphic to the predual of some TRO.

Remark 3.1. Kirchberg has proved that a subspace of the predual of von Neumann algebra is contractively complemented if it is isometrically isomorphic to the predual of some von Neumann algebra (Lemma 3.6 in [K2]). The authors conjecture that a similar statement holds for $J^{*}$-algebras. (The class of $J^{*}$-algebras (which includes $C^{*}$-algebras) is stable under the action of norm-one projections. See $[\mathbf{F R}]$.)

To prove the theorem, we need a lemma which is in itself interesting. The main idea of this lemma is the use of a suitable multiplicative domain.

Lemma 3.2. Let $V$ and $W$ be weak ${ }^{*}$-closed TROs and let $\varphi: W \rightarrow V$ be a weak*-continuous complete metric surjection. Then, there exists a sub-TRO $W_{0}$ of $W$ such that $\varphi$ maps $W_{0}$ onto $V$ and

$$
\varphi\left(x y^{*} z\right)=\varphi(x) \varphi(y)^{*} \varphi(z)
$$

for all $x, y$ and $z$ in $W$ whenever any two are in $W_{0}$. Moreover, there exists a weak ${ }^{*}$-continuous complete contraction $\theta$ from $V$ into $W$ with $\varphi \circ \theta=\mathrm{id}_{V}$.

Proof of Lemma. Since a weak*-closed TRO is an off-diagonal corner of some von Neumann algebra, we may assume that $W$ is a von Neumann algebra $R$. Let $V$ be a nondegenerate weak ${ }^{*}$-closed TRO in $\mathbb{B}(\mathcal{K}, \mathcal{H})$. We note that the weak*-closure $M$ (resp. $N$ ) of $V V^{\sharp}$ (resp. $V^{\sharp} V$ ) is a nondegenerate von Neumann algebra in $\mathbb{B}(\mathcal{H})$ (resp. $\mathbb{B}(\mathcal{K})$ ). Let $L$ be the corresponding von Neumann algebra in $\mathbb{B}(\mathcal{H} \oplus \mathcal{K})$ defined by

$$
L=\left[\begin{array}{cc}
M & V \\
V^{\sharp} & N
\end{array}\right] .
$$

We will freely identify $V$ (resp. $M, N$ ) with the corresponding subset of $L$. Let $p_{\mathcal{H}} \in L$ (resp. $p_{\mathcal{K}} \in L$ ) be the orthogonal projection onto $\mathcal{H}$ (resp $\mathcal{K}$ ). 
By the Paulsen's "off-diagonal" trick $[\mathbf{P}]$, there are unital completely positive maps $\psi: R \rightarrow \mathbb{B}(\mathcal{H})$ and $\rho: R \rightarrow \mathbb{B}(\mathcal{K})$ so that the map $\Phi: \mathbb{M}_{2}(R) \rightarrow$ $\mathbb{B}(\mathcal{H} \oplus \mathcal{K})$ defined by

$$
\Phi=\left[\begin{array}{cc}
\psi & \varphi \\
\varphi^{*} & \rho
\end{array}\right]
$$

is completely positive. Let $D \subseteq \mathbb{M}_{2}(R)$ be the multiplicative domain of $\Phi$ (cf. $[\mathbf{C}]$ ). We will prove that $\Phi(D)$ contains $V=p_{\mathcal{H}} L p_{\mathcal{K}}$.

By the comparison theorem, there exists a central projection $e \in L$ so that

$$
e p_{\mathcal{H}} \succeq e p_{\mathcal{K}} \text { and }(1-e) p_{\mathcal{H}} \preceq(1-e) p_{\mathcal{K}}
$$

Let $u \in L$ (resp. $v \in L$ ) be a partial isometry so that $u u^{*} \leq e p_{\mathcal{H}}$ and $u^{*} u=$ $e p_{\mathcal{K}}$ (resp. $v v^{*}=(1-e) p_{\mathcal{H}}$ and $\left.v^{*} v \leq(1-e) p_{\mathcal{K}}\right)$. It is clear that $u \in V$ (resp. $v \in V$ ). We note that $V=p_{\mathcal{H}} L p_{\mathcal{K}}$ is decomposed as $V=e V \oplus(1-e) V$. Since $u^{*} u=e p_{\mathcal{K}}$, we have $e V=e M u$. Since a von Neumann algebra is the norm closure of the linear span of its unitaries, $e V$ is the norm closure of the linear span of its partial isometries. Similar reasoning holds for $(1-e) V$. Hence, $V$ is the norm closure of the linear span of its partial isometries.

Fix a partial isometry $r \in V$. Observe that if $\left\{r_{i}\right\}_{i \in I}$ is a family of partial isometries in $V$ with mutually orthogonal ranges, then we have $\sum_{i \in I} r_{i} r_{i}^{*} \in$ $M$ and thus

$$
\left(p_{\mathcal{H}}-\sum_{i \in I} r_{i} r_{i}^{*}\right) V \subseteq V .
$$

By this observation, the nondegeneracy of $V$ in $\mathbb{B}(\mathcal{K}, \mathcal{H})$, and Zorn's Lemma, there exists an index set $I$ with distinguished element 1 and a family $\left\{r_{i}\right\}_{i \in I}$ of partial isometries in $V$, with mutually orthogonal ranges, such that

$$
r_{1}=r \text { and } \sum_{i \in I} r_{i} r_{i}^{*}=p_{\mathcal{H}}
$$

Enlarging $I$ if necessary, we may find a family $\left\{v_{i}\right\}_{i \in I}$ of partial isometries in $V$, with mutually orthogonal initial spaces such that

$$
v_{1}=r \text { and } \sum_{i \in I} v_{i}^{*} v_{i}=p_{\mathcal{K}} .
$$

Let us denote $\mathbb{B}\left(\ell_{2}(I)\right)$ by $\mathbb{M}_{I}$ and identify $\mathbb{M}_{I}$ with the $I \times I$ matrices. We claim that the map $\operatorname{id}_{\mathbb{M}_{I}} \otimes \varphi$ maps the closed unit ball of $\mathbb{M}_{I}(R)$ onto the closed unit ball of $\mathbb{M}_{I}(V)$. Since $\varphi$ is weak ${ }^{*}$-continuous, we have that $\varphi_{*}$ is a complete isometry from $V_{*}$ into $R_{*}$. Thus, the claim follows from the isometric identifications

$$
C B\left(V_{*}, \mathbb{M}_{I}\right)=\mathbb{M}_{I}(V) \text { and } C B\left(R_{*}, \mathbb{M}_{I}\right)=\mathbb{M}_{I}(R)
$$

(cf. $[\mathbf{E R} 1]$ ) and the injectivity of $\mathbb{M}_{I}$.

Let $x \in \mathbb{M}_{I}(V)$ be the element defined by:

(1) $x_{1 i}=r_{i}$ for $i \in I$, 
(2) $x_{i 1}=v_{i}$ for $i \in I$, and

(3) $x_{i j}=0$ if $i \neq 1$ and $j \neq 1$.

We remark that in the case $I=\mathbb{N}$, the matrix $x$ looks like

$$
x=\left[\begin{array}{ccccc}
r & r_{2} & r_{3} & r_{4} & \ldots \\
v_{2} & 0 & 0 & 0 & \\
v_{3} & 0 & 0 & & \\
v_{4} & 0 & & & \\
\vdots & & & &
\end{array}\right] .
$$

By our choices of $r_{i} \mathrm{~s}$ and $v_{i} \mathrm{~s}$, we have that $\|x\|=1$. By the previous remark, we may find $\widetilde{x} \in \mathbb{M}_{I}(R)$ with $\|\widetilde{x}\|=1$ such that $\operatorname{id}_{\mathbb{M}_{I}} \otimes \varphi(\widetilde{x})=x$. Let $\widetilde{r} \in R$ be the "1-1 entry" of $\widetilde{x}$. Clearly, we have $\varphi(\widetilde{r})=r$. Let us define $s \in \mathbb{M}_{2}(R)$ by

$$
s=\left[\begin{array}{cc}
0 & \widetilde{r} \\
0 & 0
\end{array}\right] .
$$

We will prove that $s \in D$. By the Choi-Schwarz inequality, we have

$$
\left[\begin{array}{cc}
\psi\left(\widetilde{r} \widetilde{r}^{*}\right) & 0 \\
0 & 0
\end{array}\right]=\Phi\left(s s^{*}\right) \geq \Phi(s) \Phi(s)^{*}=\left[\begin{array}{cc}
r r^{*} & 0 \\
0 & 0
\end{array}\right] .
$$

Let $t=\psi\left(\widetilde{r} r^{*}\right)$. We note that $0 \leq r r^{*} \leq t \leq 1$ and $r r^{*}$ is a projection. If $t \neq r r^{*}$, then there is $i \in I \backslash\{1\}$ such that $r_{i} r_{i}^{*} t \neq 0$, but this contradicts the inequality

$$
1 \geq \psi\left(\widetilde{r} \widetilde{r}^{*}+\widetilde{x}_{1 i} \widetilde{x}_{1 i}^{*}\right) \geq t+r_{i} r_{i}^{*} .
$$

Therefore, we have $t=r r^{*}$ or equivalently, $s$ is in the left multiplicative domain of $\Phi$. Similarly, we can show that $s$ is in the right multiplicative domain of $\Phi$. Consequently, we have $s \in D$. But $V$ is spanned by its partial isometries. Hence,

$$
V=p_{\mathcal{H}} L p_{\mathcal{K}} \subseteq \Phi(D) .
$$

Finally, let $W_{0}$ be the sub-TRO of $R$ corresponding to the "1-2 corner" of $D$. (It should be noted that $p_{\mathcal{H}}, p_{\mathcal{K}} \in D$.) By our assumption, $\varphi$ maps $W$, and hence $W_{0}$, into $V$. Therefore, by the argument that we have just finished, $\varphi$ maps $W_{0}$ onto $V$. It is easy to check that $W_{0}$ has the other desired properties. This completes the proof of the first part.

We now prove the second part. $W_{0}$ is weak ${ }^{*}$-closed and $\left.\varphi\right|_{W_{0}}$ is a weak*closed TRO-homomorphism a from $W_{0}$ onto $V$. Since a weak ${ }^{*}$-continuous TRO-homomorphism splits (cf. [EOR]) as in the von Neumann algebra case, we are done.

Remark 3.3. We note that, as in the von Neumann algebra case, more than one $\theta$ can satisfy the statement of Lemma 3.2, but there must be a unique minimal one. 
Proof of Theorem 1.1. Let $X$ be a subspace of the predual $W_{*}$ of a TRO $W$. If $X$ is completely 1-complemented in $W_{*}$, then $X^{*}$ is completely isometric to a completely 1-complemented subspace of $W$ and thus completely isometric to a TRO, by Youngson's theorem $[\mathbf{Y}]$.

We now prove the "if" part. Suppose that $V=X^{*}$ is a TRO. By [EOR], $V$ is a weak*-closed TRO. Let $\varphi: W \rightarrow V$ be the dual map of a complete isometry from $V_{*}=X$ into $W_{*}$. By Lemma 3.2, there exists a weak*continuous lifting $\theta: V \rightarrow W$ of $\varphi$. The map $\varphi_{*} \circ \theta_{*}$ is the desired projection.

Proof of Corollary 1.2. Suppose that $R_{1}$ is an injective von Neumann algebra, and $R_{2}$ is a von Neumann algebra such that there is a complete isometry $i:\left(R_{2}\right)_{*} \rightarrow\left(R_{1}\right)_{*}$. Then by Theorem 1.1 , there is a completely contractive projection $p$ on $\left(R_{1}\right)_{*}$ whose image is $i\left(\left(R_{2}\right)_{*}\right)$. Hence, $p^{*}$ is a completely contractive projection on $R_{1}$ whose image is completely isometric to $R_{2}$. Therefore, since $R_{1}$ is injective, so is $R_{2}$.

\section{Preduals of separable injective von Neumann algebras.}

We give an operator space characterization of the preduals of separable injective von Neumann algebras. This is a generalization of a classical result characterizing $L_{1}$ spaces (mentioned in the introduction).

The bulk of the work in Theorem 1.7 was done in [ER2]. Our contribution is in the implication $(3) \Rightarrow(1)$ of the theorem. The key technique in our proof is a deep result from [EOR], which states that any dual injective operator space has the form $p R q$, where $R$ is an injective von Neumann, and $p, q$ are projections in $R$. For the convenience of the reader, we will repeat the necessary definitions before giving the argument.

Definition 4.1. We say that an operator space $\mathrm{X}$ is a rigid $\mathcal{O} \mathcal{L}_{1,1+}$ space if given elements $\omega_{1}, \ldots, \omega_{s} \in X$ and $\epsilon>0$, we can find positive integers $n_{i}$ and a complete isometry

$$
S: \mathbb{T}_{n_{1}} \oplus \cdots \oplus \mathbb{T}_{n_{k}} \rightarrow X
$$

with

$$
\operatorname{dist}\left(\omega_{j}, S\left(\mathbb{T}_{n_{1}} \oplus \cdots \oplus \mathbb{T}_{n_{k}}\right)\right)<\epsilon
$$

for all $j$.

Definition 4.2. Let us suppose that $\lambda>1$. We say that an operator space $X$ is an $\mathcal{O} \mathcal{L}_{1, \lambda}$ space if for every finite dimensional subspace $E$ of $X$, there exists a finite dimensional subspace $F$ with $E \subseteq F \subseteq X$ such that

$$
d_{c b}\left(F, \mathbb{T}_{n_{1}} \oplus \cdots \oplus \mathbb{T}_{n_{k}}\right)<\lambda
$$


for some integers $n_{i}$. We are using Pisier's operator space analogue of the Banach-Mazur distance:

$$
d_{c b}(X, Y)=_{\mathrm{df}} \inf \left\{\|T\|_{c b}\left\|T^{-1}\right\|_{c b}: T: X \rightarrow Y \text { a linear homeomorphism }\right\} .
$$

Definition 4.3. We say that an operator space $X$ is an $\mathcal{O} \mathcal{L}_{1,1+}$ space if it is $\mathcal{O} \mathcal{L}_{1, \lambda}$ for every $\lambda>1$.

Theorem 1.7. Let $X$ be a separable operator space. Then the following conditions on $X$ are equivalent:

(1) $X$ is completely isometric to the predual of an injective von Neumann algebra.

(2) $X$ is rigid $\mathcal{O} \mathcal{L}_{1,1+}$.

(3) $X$ is $\mathcal{O} \mathcal{L}_{1,1+}$.

Proof. The implications (1) $\Rightarrow(2)$ and $(2) \Rightarrow(3)$ follow from arguments in [ER2]. We now prove $(3) \Rightarrow(1)$.

Suppose that $X$ is a separable $\mathcal{O} \mathcal{L}_{1,1+}$ space. By [EOR], there is a separable injective von Neumann algebra $R$, and projections $p, q \in R$ such that $X$ is completely isometric to $q R_{*} p$. We may assume that $R$ acts on a Hilbert space $\mathcal{H}$ such that $\overline{q R p \mathcal{H}}=q \mathcal{H}, \overline{p R q \mathcal{H}}=p \mathcal{H}$, and every $\rho \in R_{*}$ has the form $\rho()=.\langle. h \mid k\rangle$ for some vectors $h, k \in \mathcal{H}$.

Since $X$ is an $\mathcal{O} \mathcal{L}_{1,1+}$ space, let $X=\overline{\bigcup_{m=1}^{\infty} X_{m}}$, where for all $\mathrm{m}$,

a) $X_{m} \subseteq X_{m+1}$, and

b) there are integers $n_{i}$, dependent on $m$, such that

$$
d_{c b}\left(X_{m}, \mathbb{T}_{n_{1}} \oplus \cdots \oplus \mathbb{T}_{n_{k}}\right)<1+1 / m ;
$$

in particular, let

$$
i_{m}: \mathbb{T}_{n_{1}} \oplus \cdots \oplus \mathbb{T}_{n_{k}} \rightarrow X_{m}
$$

be an isomorphism onto $X_{m}$ such that $\left\|i_{m}\right\|_{c b} \leq 1$ and $\left\|i_{m}^{-1}\right\|_{c b} \leq$ $1+1 / m$.

For all $m$, thinking of $i_{m}$ as a map into $X$, consider the dual map

$$
\phi_{m}={ }_{\mathrm{df}} i_{m}^{*}: X^{*} \rightarrow i_{m}^{-1}\left(X_{m}\right)^{*} .
$$

Note that $i_{m}^{-1}\left(X_{m}\right)^{*}$ is a finite dimensional $\mathrm{C}^{*}$ algebra, and thus has a unit. Hence, for all $m$, choose $x_{m} \in X^{*}$ so that

$$
\left\|x_{m}\right\| \leq 1+1 / m \text { and } \phi_{m}\left(x_{m}\right)=1 \text {. }
$$

We will show that $l\left(x_{m}\right) \rightarrow p$ and $r\left(x_{m}\right) \rightarrow q$ in SOT, and thus $p$ is Murray von Neumann equivalent to $q$.

Claim 1. There is a sequence $\left\{\epsilon_{m}\right\}_{m=1}^{\infty}$ of positive real numbers such that:

i) $\epsilon_{m} \rightarrow 0$ as $m \rightarrow \infty$, and

ii) for every $y \in V$ with $\|y\| \leq 1, l\left(x_{m}\right) \perp l(y) \Rightarrow\left\|\phi_{m}(y)\right\| \leq \epsilon_{m}$. 
Suppose that $y \in V$ is such that $\|y\| \leq 1$ and $l\left(x_{m}\right) \perp l(y)$. Then

$$
\begin{aligned}
\left\|\left[\phi_{m}\left(x_{m}\right) \phi_{m}(y)\right]\right\| & \leq\left\|\left(\phi_{m}\right)_{2}\left(\left[\begin{array}{cc}
x_{m} & y \\
0 & 0
\end{array}\right]\right)\right\| \\
& \leq\left\|\left(\phi_{m}\right)_{2}\right\|\left\|x_{m} x_{m}^{*}+y y^{*}\right\|^{1 / 2} \\
& \leq 1+1 / m, \quad \text { since } l(y) \perp l\left(x_{m}\right) .
\end{aligned}
$$

But we also have that

$$
\begin{aligned}
\left\|\left[\begin{array}{ll}
\phi_{m}\left(x_{m}\right) & \phi_{m}(y)
\end{array}\right]\right\| & =\left\|\phi_{m}\left(x_{m}\right) \phi_{m}\left(x_{m}\right)^{*}+\phi_{m}(y) \phi_{m}(y)^{*}\right\|^{1 / 2} \\
& =\left\|1+\phi_{m}(y) \phi_{m}(y)^{*}\right\|^{1 / 2}
\end{aligned}
$$

Hence,

$$
\left\|1+\phi_{m}(y) \phi_{m}(y)^{*}\right\|^{1 / 2} \leq 1+1 / m .
$$

It follows that we can choose a sequence $\left\{\epsilon_{m}\right\}$ of positive reals, independent of $y$, which satisfies the statement of Claim 1 .

Claim 2. $l\left(x_{m}\right) \rightarrow p$ in SOT in $R$.

Let $\omega()=.\langle. a k \mid h\rangle \in p R_{*} p$, where $k \in q \mathcal{H}, h \in p \mathcal{H}, a \in p R q$; and let $\rho()=.\langle. k \mid h\rangle \in q R_{*} p$. For all $\mathrm{m}$, let $f_{m} \in i_{m}^{-1}\left(X_{m}\right)$ with $\left\|f_{m}\right\| \leq 2\|\rho\|$ such that $\left\|i_{m}\left(f_{m}\right)-\rho\right\| \leq g(m)$, and such that $g(m) \rightarrow 0$, as $m \rightarrow \infty$. Hence,

$$
\begin{aligned}
\left|\omega\left(p-l\left(x_{m}\right)\right)\right|= & \left|\left\langle\left(p-l\left(x_{m}\right)\right) a k \mid h\right\rangle\right| \\
= & \left|\rho\left(\left(p-l\left(x_{m}\right)\right) a\right)\right| \\
\leq & \left|i_{m}\left(f_{m}\right)\left(\left(p-l\left(x_{m}\right)\right) a\right)\right|+g(m)\|a\| \\
= & \left|f_{m}\left(i_{m}^{*}\left(\left(p-l\left(x_{m}\right)\right) a\right)\right)\right|+g(m)\|a\| \\
\leq & 2\|\rho\| \epsilon_{m}+g(m)\|a\| \rightarrow 0 \\
& \text { as } m \rightarrow \infty .
\end{aligned}
$$

But functionals of the form $\omega$ are dense in $p R_{*} p$. Thus, $l\left(x_{m}\right) \rightarrow p$ in WOT in $R$; and hence, since the $l\left(x_{m}\right)$ s are projections smaller than $p, l\left(x_{m}\right) \rightarrow p$ in $\mathrm{SOT}$ in $R$.

Claim 3. $r\left(x_{m}\right) \rightarrow q$ in SOT in $R$.

The proof of this claim is similar to that of Claim 2 .

From Claims 2, 3, and [Tak] p. 344 question 3, the projections $p$ and $q$ are Murray von Neumann equivalent in $R$. Hence, $p R q$ is completely isometric to $p R p$, an injective von Neumann algebra. Hence, $X$ is completely isometric to the predual of an injective von Neumann algebra. 


\section{Finite dimensional completely 1-complemented subspaces.}

The goal of this section is to concretely analyze the spaces $X$ of Theorem 1.1, when those spaces are finite dimensional. The main result of this section is:

Proposition 5.1. Suppose that we have positive integers $n_{l}, m_{l}$ for $1 \leq l \leq$ k. Let

$$
S: \mathbb{T}_{n_{1}, m_{1}} \oplus \mathbb{T}_{n_{2}, m_{2}} \oplus \cdots \oplus \mathbb{T}_{n_{k}, m_{k}} \rightarrow R_{*}
$$

be a complete isometry. Then there exists partial isometries $\left\{\left\{u_{j}^{l}\right\}_{j=1}^{m_{l}}\right\}_{l=1}^{k}$ and $\left\{\left\{w_{i}^{l}\right\}_{i=1}^{n_{l}}\right\}_{l=1}^{k}$ such that:

(1) $r\left(u_{j}^{l}\right)=r\left(S\left(e_{1,1}^{l}\right)\right)$ and $r\left(w_{i}^{l}\right)=l\left(S\left(e_{1,1}^{l}\right)\right)$ for all $i, j, l$,

(2) the projections $\left\{\left\{l\left(u_{j}^{l}\right)\right\}_{j=1}^{m_{l}}\right\}_{l=1}^{k}$ are pairwise orthogonal,

(3) the projections $\left\{\left\{l\left(w_{i}^{l}\right)\right\}_{i=1}^{n_{l}}\right\}_{l=1}^{k}$ are pairwise orthogonal,

(4) $S\left(e_{i, j}^{l}\right)=w_{i}^{l} S\left(e_{1,1}^{l}\right)\left(u_{j}^{l}\right)^{*}$ for all $i, j, l$.

Proposition 5.1, together with the following observation, characterizes the finite dimensional completely 1-complemented subspaces of a von Neumann algebra predual $R_{*}$.

Proposition 5.2. Let $R$ be a von Neumann algebra. Let $\left\{\omega^{l}\right\}_{l=1}^{k}$ be a sequence of pairwise orthogonal elements of $R_{*}$ with $\left\|\omega^{l}\right\|=1$ for all $l$. Suppose that there exist partial isometries $\left\{\left\{u_{j}^{l}\right\}_{j=1}^{m_{l}}\right\}_{l=1}^{k}$ and $\left\{\left\{w_{i}^{l}\right\}_{i=1}^{n_{l}}\right\}_{l=1}^{k}$ such that:

(1) $r\left(u_{j}^{l}\right)=r\left(\omega^{l}\right)$ and $r\left(w_{i}^{l}\right)=l\left(\omega^{l}\right)$ for all $i, j, l$,

(2) the projections $\left\{\left\{l\left(u_{j}^{l}\right)\right\}_{j=1}^{m_{l}}\right\}_{l=1}^{k}$ are pairwise orthogonal,

(3) the projections $\left\{\left\{l\left(w_{i}^{l}\right)\right\}_{i=1}^{n_{l}}\right\}_{l=1}^{k}$ are pairwise orthogonal.

Then the linear map $\mathbb{T}_{n_{1}, m_{1}} \oplus \cdots \oplus \mathbb{T}_{n_{k}, m_{k}} \rightarrow R_{*}$ given by

$$
e_{i, j}^{l} \mapsto w_{i}^{l} \omega^{l}\left(u_{j}^{l}\right)^{*}
$$

is a complete isometry.

Proposition 5.1 will also yield an alternate proof of Theorem 1.1, when $X$ is finite dimensional. This is the content of Propositions 5.6 and 5.7.

We now list two technical propositions whose proofs can be found in [Yead]. The first, Proposition 5.3, gives a condition for when two normal linear functionals are orthogonal. The condition is the same as the usual one for positive normal linear functionals:

Proposition 5.3. Let $R$ be a von Neumann algebra. Let $\omega, \gamma$ be elements of $R_{*}$. Then $\omega \perp \gamma$ if and only if $\|\omega \pm \gamma\|=\|\omega\|+\|\gamma\|$.

Proposition 5.4 is the key technique of this section.

Proposition 5.4 (Yeadon). Let $R$ be a von Neumann algebra. Let $S$ : $\mathbb{T}_{n} \rightarrow R_{*}$ be an isometry. Let $S(1)=w|S(1)|$ be the polar decomposition of $S(1)$. Then there is a Jordan *-embedding $J: \mathbb{M}_{n} \rightarrow R$ such that: 
(1) $J(e)=\operatorname{supp}(|S(e)|)$ for every projection e in $\mathbb{M}_{n}$,

(2) $J(e)|S(1)|=|S(1)| J(e)=S(e)$ for every projection e in $\mathbb{M}_{n}$,

(3) $S(x)=S(1) J(x)$ for all $x$ in $\mathbb{T}_{n}\left(=\mathbb{M}_{n}\right.$ setwise $)$.

In particular, $J(1)=\operatorname{supp}(|S(1)|)=w^{*} w$ and $J(x)|S(1)|=|S(1)| J(x)$ for all $x$ in $\mathbb{T}_{n}$.

(Here "supp $(|S(1)|)$ " denotes the support projection of the positive normal functional $|S(1)|)$.

Changes in the arguments of [Yead] and [K2] Lemma 3.6 will yield a proof that, in Proposition 5.4, that $S$ is a complete isometry implies that $J$ is a *-isomorphism. One can also prove this directly from Proposition 5.4 by a messy argument. Since we will always be working with complete isometries, we make the following assumption:

For the rest of this paper, $J$ will always be $a^{*}$-isomorphism.

For the convenience of the reader, we now give a proof of Proposition 5.4 for the case where $S$ is a complete isometry (and hence $J$ a ${ }^{*}$-isomorphism). The proof will follow the ideas of [K2] Lemma 3.6.

Proof of Proposition 5.4 for $S$ a complete isometry. Since $S$ is a complete isometry, the dual map $S^{*}: R \rightarrow \mathbb{M}_{n}$ is a $w^{*}$-continuous complete metric surjection. Let

$$
S(1)=w|S(1)|
$$

be the polar decomposition of $S(1)$. Let $\Phi: R \rightarrow \mathbb{M}_{n}$ be the map given by

$$
\Phi(x)={ }_{\mathrm{df}} S^{*}\left(x w^{*}\right) \text {, for all } x \in R .
$$

$\Phi$ is a unital, $w^{*}$-continuous, completely positive, complete metric surjection. Let $D$ be the multiplicative domain of $\Phi$. We will show that $\Phi(D)$ contains $\mathbb{M}_{n}$.

Suppose that $e \in \mathbb{M}_{n}$ is a projection. Let $x \in R$ with $x^{*}=x,\|x\| \leq 1$ be such that $\Phi(x)=1-2 e$. Therefore, by the Choi-Schwarz inequality

$$
1 \leq \Phi\left(x^{2}\right) \leq \Phi(x)^{2}=(1-2 e)^{2}=1 .
$$

Hence $x \in D$ and $e \in \Phi(D)$. Therefore, since $\mathbb{M}_{n}$ is spanned by its projections,

$$
\mathbb{M}_{n}=\Phi(D)
$$

as required.

Now $D$ is a von Neumann algebra and the restriction map $\Phi: D \rightarrow \mathbb{M}_{n}$ is a $w^{*}$-continuous ${ }^{*}$-homomorphism onto $\mathbb{M}_{n}$. Therefore, let $J: \mathbb{M}_{n} \rightarrow D$ be the minimal ${ }^{*}$-homomorphism such that

$$
\Phi \circ J=\mathrm{id}_{\mathbb{M}_{n}},
$$


where $\operatorname{id}_{\mathbb{M}_{n}}$ is the identity map on $\mathbb{M}_{n}$. Hence,

$$
S^{*}\left(J(a) w^{*}\right)=a
$$

for all $a \in \mathbb{M}_{n}$. Hence

$$
S(b)\left(J(a) w^{*}\right)=\operatorname{tr}(a b)
$$

for all $a, b \in \mathbb{M}_{n}$, where tr is the trace on $\mathbb{M}_{n}$ and we identify $\mathbb{T}_{n}$ with $\mathbb{M}_{n}$ in the natural way.

Hence, for every projection $e \in \mathbb{M}_{n}$, we have that

$$
\begin{aligned}
|S(e)|(J(e)) & =S(e)\left(J(e) w^{*}\right) \\
& =\operatorname{tr}(e) \\
& =\|e\|_{1} \\
& =\|S(e)\|_{1}
\end{aligned}
$$

Here \|\|$_{1}$ is the norm on $\mathbb{T}_{n}$.

Here \|\|$_{1}$ is the norm on $R_{*}$.

Also, $|S(e)|(J(1-e))=0$. Therefore, by the minimality of $J$,

$$
J(e)=\operatorname{supp}(|S(e)|)
$$

as required.

The other clauses in the proposition follow from this and applications of Proposition 5.3.

Using Propositions 5.3 and 5.4, we now analyze the "square" complete isometries $S: \mathbb{T}_{n} \rightarrow R_{*}$.

Lemma 5.5. Let $R$ be a von Neumann algebra. Let $S: \mathbb{T}_{n} \rightarrow R_{*}$ be complete isometry. Let $J$ be the Jordan *-isomorphism from Proposition 5.4. Then for all $i, j$, we have that:

(1) $\left|S\left(e_{i, j}\right)\right|=|S(1)| J\left(e_{j, j}\right)=\left|S\left(e_{j, j}\right)\right|$,

(2) $S\left(e_{i . j}\right)=\left(w J\left(e_{i, j}\right)\right)\left(|S(1)| J\left(e_{j, j}\right)\right)$ is the polar decomposition of $S\left(e_{i, j}\right)$,

(3) $v\left(S\left(e_{i, j}\right)\right)=w J\left(e_{i, j}\right)$, where $v\left(S\left(e_{i, j}\right)\right)$ is the partial isometry from the polar decomposition of $S\left(e_{i, j}\right)$,

and for $i \neq k$ and $j \neq l$, we have that:

(4) $l\left(S\left(e_{i, j}\right)\right)=l\left(S\left(e_{i, l}\right)\right)$,

(5) $r\left(S\left(e_{i, j}\right)\right) \perp r\left(S\left(e_{i, l}\right)\right)$,

(6) $l\left(S\left(e_{i, j}\right)\right) \perp l\left(S\left(e_{k, j}\right)\right)$,

(7) $r\left(S\left(e_{i, j}\right)\right)=r\left(S\left(e_{k, j}\right)\right)$,

and for $1 \leq i, j \leq n$, if we let $u_{j}==_{\mathrm{df}} v\left(S\left(e_{1, j}\right)\right)^{*} v\left(S\left(e_{1,1}\right)\right)$ and $w_{i}=_{\mathrm{df}}$ $v\left(S\left(e_{i, 1}\right)\right) v\left(S\left(e_{1,1}\right)\right)^{*}$, then:

(8) $r\left(u_{j}\right)=r\left(S\left(e_{1,1}\right)\right)$ and $r\left(w_{i}\right)=l\left(S\left(e_{1,1}\right)\right)$ for all $i, j$,

(9) the projections $\left\{l\left(u_{j}\right): 1 \leq j \leq n\right\}$ are pairwise orthogonal,

(10) the projections $\left\{l\left(w_{i}\right): 1 \leq i \leq n\right\}$ are pairwise orthogonal,

(11) $S\left(e_{i, j}\right)=w_{i} S\left(e_{1,1}\right) u_{j}^{*}$, for all $i, j$. 
Proof. Statements (1), (2) and (3) together: Repeatedly applying Proposition 5.4,

$$
\begin{aligned}
\left(w J\left(e_{i, j}\right)\right)\left(|S(1)| J\left(e_{j, j}\right)\right) & =w|S(1)| J\left(e_{i, j}\right) J\left(e_{j, j}\right) \\
& =w S(1) J\left(e_{i, j}\right) \\
& =S\left(e_{i, j}\right) .
\end{aligned}
$$

But $|S(1)| J\left(e_{j, j}\right)=\left|S\left(e_{j, j}\right)\right| \geq 0$, with support projection $J\left(e_{j, j}\right)$. Also,

$$
\begin{aligned}
\left(w J\left(e_{i, j}\right)\right)^{*}\left(w J\left(e_{i, j}\right)\right) & =J\left(e_{i, j}\right)^{*} w^{*} w J\left(e_{i, j}\right) \\
& =J\left(e_{j, i}\right) J(1) J\left(e_{i, j}\right) \\
& =J\left(e_{j, j}\right),
\end{aligned}
$$

which is, by Proposition 5.4, the support projection of the positive normal linear functional $|S(1)| J\left(e_{j, j}\right)=\left|S\left(e_{j, j}\right)\right|$. Hence, $w J\left(e_{i, j}\right)$ is a partial isometry, and by the uniqueness of polar decomposition, Statements (1), (2) and (3) follow.

Statements (4) and (6) together: Applying Proposition 5.4 and Statement (3) (which we have just proved),

$$
\begin{aligned}
l\left(S\left(e_{i, j}\right)\right) & =\left(w J\left(e_{i, j}\right)\right)\left(w J\left(e_{i, j}\right)\right)^{*} \\
& =w J\left(e_{i, j}\right) J\left(e_{i, j}\right)^{*} w^{*} \\
& =w J\left(e_{i, j} e_{j, i}\right) w^{*} \\
& =w J\left(e_{i, i}\right) w^{*} \\
& =\left(w J\left(e_{i, i}\right)\right)\left(w J\left(e_{i, i}\right)\right)^{*} \\
& =v\left(S\left(e_{i, i}\right)\right) v\left(S\left(e_{i, i}\right)\right)^{*} \\
& =l\left(S\left(e_{i, i}\right)\right) .
\end{aligned}
$$

Hence, $l\left(S\left(e_{i, j}\right)\right)=l\left(S\left(e_{i, i}\right)\right)$ for all $i, j$. Hence, $l\left(S\left(e_{i, j}\right)\right)=l\left(S\left(e_{i, i}\right)\right)=$ $l\left(S\left(e_{i, k}\right)\right)$ for all $i$, and for all $j \neq k$.

And also, from this and Proposition 5.3,

$$
l\left(S\left(e_{i, j}\right)\right)=l\left(S\left(e_{i, i}\right)\right) \perp l\left(S\left(e_{k, k}\right)\right)=l\left(S\left(e_{k, j}\right)\right),
$$

for all $j$, and for all $i \neq k$.

Statements (5) and (7) together: The proof is similar to that of (4) and (6) together.

Statements (8)-(10): These follow from Statements (4)-(7). 
Statement (11): Applying Proposition 5.4 and Statement (3) again,

$$
\begin{aligned}
w_{i} S\left(e_{1,1}\right) u_{j}^{*} & =\left(v\left(S\left(e_{i, 1}\right)\right) v\left(S\left(e_{1,1}\right)\right)^{*}\right) S\left(e_{1,1}\right)\left(v\left(S\left(e_{1,1}\right)\right)^{*} v\left(S\left(e_{1, j}\right)\right)\right) \\
& =\left(w J\left(e_{i, 1}\right) J\left(e_{1,1}\right) w^{*}\right)\left(w|S(1)| J\left(e_{1,1}\right)\right)\left(J\left(e_{1,1}\right) w^{*} w J\left(e_{1, j}\right)\right) \\
& =w J\left(e_{i, 1}\right) J(1)|S(1)| J\left(e_{1,1}\right) J(1) J\left(e_{1, j}\right) \\
& =w J\left(e_{i, 1}\right)|S(1)| J\left(e_{1, j}\right) \\
& =w|S(1)| J\left(e_{i, j}\right) \\
& =S\left(e_{i, j}\right) .
\end{aligned}
$$

We now prove Proposition 5.1. The strategy of the proof is to apply Lemma 5.5 to "square subspaces" of $\mathbb{T}_{n_{1}, m_{1}} \oplus \cdots \oplus \mathbb{T}_{n_{k}, m_{k}}$, when $n_{l}, m_{l} \geq 2$.

Proof of Proposition 5.1. First, we deal with the case where $n_{l}, m_{l} \geq 2$ for all $l$. Fix $l, 1 \leq l \leq k$. For $i, j \geq 2$, apply Lemma 5.5 to the complete isometry from $\mathbb{T}_{2}$ into $R_{*}$ given by mapping:

$$
\begin{aligned}
e_{1,1} & \mapsto S\left(e_{1,1}^{l}\right) \\
e_{1,2} & \mapsto S\left(e_{1, j}^{l}\right) \\
e_{2,1} & \mapsto S\left(e_{i, 1}^{l}\right) \\
e_{2,2} & \mapsto S\left(e_{i, j}^{l}\right) .
\end{aligned}
$$

If we repeat this process for all $l, 1 \leq l \leq k$, and for all $i, j, 1 \leq i, j \leq n_{l}$, then by Lemma 5.5 and Proposition 5.3, we get partial isometries $\left\{u_{j}^{l}\right\}$ and $\left\{w_{i}^{l}\right\}$ in $R$ such that:

a) $r\left(u_{j}^{l}\right)=r\left(S\left(e_{1,1}^{l}\right)\right)$ and $r\left(w_{i}^{l}\right)=l\left(S\left(e_{1,1}^{l}\right)\right)$ for all $i, j, l$,

b) $l\left(u_{1}^{l}\right) \perp l\left(u_{j}^{l}\right)$ and $l\left(w_{1}^{l}\right) \perp l\left(w_{i}^{l}\right)$ for all $l$, and for $i \neq 1, j \neq 1$,

c) $l\left(u_{j}^{l}\right) \perp l\left(u_{s}^{r}\right)$ and $l\left(w_{i}^{l}\right) \perp l\left(w_{t}^{r}\right)$ for all $j, s, i, t$, and for $l \neq r$, and

d) $S\left(e_{i, j}^{l}\right)=w_{i}^{l} S\left(e_{1,1}^{l}\right)\left(u_{j}^{l}\right)^{*}$ for all $i, j, l$.

Thus, to finish the proof of the special case, it suffices to show that for all $l, 1 \leq l \leq k$,

i) $l\left(u_{a}^{l}\right) \perp l\left(u_{b}^{l}\right)$ for $a \neq b$ and $a, b \geq 2$, and

ii) $l\left(w_{c}^{l}\right) \perp l\left(w_{d}^{l}\right)$ for $c \neq d$ and $c, d \geq 2$.

Statement i): We may assume that $a<b$. Firstly, note that by definition, we have that

$$
\begin{aligned}
l\left(u_{a}^{l}\right) & =l\left(v\left(S\left(e_{1, a}^{l}\right)\right)^{*} v\left(S\left(e_{1,1}^{l}\right)\right)\right) \\
& =l\left(v\left(S\left(e_{1, a}^{l}\right)\right)^{*}\right) \text { by Lemma } 5.5 \\
& =r\left(S\left(e_{1, a}^{l}\right)\right) .
\end{aligned}
$$

Next, if we consider the complete isometry $\mathbb{T}_{2} \rightarrow R_{*}$ given be the maps 


$$
\begin{aligned}
& e_{1,1} \mapsto S\left(e_{1, a}^{l}\right) \\
& e_{1,2} \mapsto S\left(e_{1, b}^{l}\right) \\
& e_{2,1} \mapsto S\left(e_{2, a}^{l}\right) \\
& e_{2,2} \mapsto S\left(e_{2, b}^{l}\right)
\end{aligned}
$$

then, by Lemma 5.5, it follows that $r\left(S\left(e_{1, a}^{l}\right)\right) \perp r\left(S\left(e_{1, b}^{l}\right)\right)$; and hence, $l\left(u_{a}^{l}\right) \perp l\left(u_{b}^{l}\right)$, as required.

Statement ii): The proof is similar to that of Statement i).

For the general case where $n_{l}, m_{l}$ are arbitrary positive integers (not necessarily $\geq 2$ ) for all $l$, consider the complete isometry

$$
S_{2}: \mathbb{T}_{2} \otimes\left(\mathbb{T}_{n_{1}, m_{1}} \oplus \cdots \oplus \mathbb{T}_{n_{k}, m_{k}}\right) \rightarrow \mathbb{T}_{2} \otimes R_{*}
$$

By the previous case, we get partial isometries in $\mathbb{M}_{2}(R)$. From these, we can extract partial isometries in $R$, which are the required ones.

We note that under the same hypothesis as Proposition 5.1, we have that

(1) $v\left(S\left(e_{i, j}^{l}\right)\right)=w_{i}^{l} v\left(S\left(e_{1,1}^{l}\right)\right)\left(u_{j}^{l}\right)^{*}$, where $v\left(S\left(e_{a, b}^{l}\right)\right)$ is the partial isometry from the polar decomposition of $S\left(e_{a, b}^{l}\right)$, and

(2) $r\left(w_{i}^{l}\right)=l\left(S\left(e_{1,1}^{l}\right)\right)$, and $r\left(u_{j}^{l}\right)=r\left(S\left(e_{1,1}^{l}\right)\right)$ for all $i, j, l$.

This follows from Proposition 5.1. Hence, the space

$$
V={ }_{\mathrm{df}} \operatorname{Span}\left\{v\left(S\left(e_{i, j}^{l}\right)\right)^{*}\right\}
$$

is a finite dimensional sub-TRO of the von Neumann algebra $R$. Indeed, the map $\mathbb{M}_{n_{1}, m_{1}} \oplus \cdots \oplus \mathbb{M}_{n_{k}, m_{k}} \rightarrow V$, given by

$$
e_{i, j}^{l} \mapsto v\left(S\left(e_{i, j}^{l}\right)\right)^{*}
$$

is a TRO-isomorphism onto $V$, and hence, is a complete isometry. This observation allows us to now give a concrete example of the completely contractive projections in Theorem 1.1. (What we give will arise from the predual of a TRO-homomorphism $\theta$ from Lemma 3.2.)

Suppose that $n_{l}, m_{l}$ are positive integers for $1 \leq l \leq k$. Suppose that $S: \mathbb{T}_{n_{1}, m_{1}} \oplus \ldots \mathbb{T}_{n_{k}, m_{k}} \rightarrow R_{*}$ is a complete isometry. Let $X=_{\mathrm{df}} S\left[\mathbb{T}_{n_{1}, m_{1}} \oplus\right.$ $\left.\cdots \oplus \mathbb{T}_{n_{k}, m_{k}}\right]$ be the image of S. Let $p: R_{*} \rightarrow X$ be given by

$$
p(\omega)={ }_{\mathrm{df}} \sum_{l=1}^{k} \sum_{1 \leq i \leq n_{l}, 1 \leq j \leq m_{l}}\left\langle\omega \mid v\left(S\left(e_{i, j}^{l}\right)\right)\right\rangle S\left(e_{i, j}^{l}\right),
$$

for all $\omega \in R_{*}$. (Here $v\left(S\left(e_{i, j}^{l}\right)\right)$ is the partial isometry from the polar decomposition of $S\left(e_{i, j}^{l}\right)$.)

Proposition 5.6. $p$ is a completely contractive projection onto $X$. 
Proof. First, by Proposition 5.1, we have that

$$
\begin{aligned}
p\left(S\left(e_{r, s}^{t}\right)\right) & =\sum_{i, j, l}\left\langle S\left(e_{r, s}^{t}\right) \mid v\left(S\left(e_{i, j}^{l}\right)\right)\right\rangle S\left(e_{i, j}^{l}\right) \\
& =S\left(e_{r, s}^{t}\right) \text { for all } r, s, t .
\end{aligned}
$$

Hence, $p$ is a projection onto $X$. But the dual map $\left(S^{-1} \circ p\right)^{*}: \mathbb{M}_{n_{1}, m_{1}} \oplus$ $\cdots \oplus \mathbb{M}_{n_{k}, m_{k}} \rightarrow R$ is the TRO-isomorphism given by the maps $e_{i, j}^{l} \mapsto$ $v\left(S\left(e_{j, i}^{l}\right)\right)^{*}$. Hence, $p$ must be completely contractive; and in particular, $p$ is an example of the type of projection in Theorem 1.1.

Proposition 5.6, together with the next proposition, show that the $f$ nite dimensional completely 1-complemented subspaces of a von Neumann algebra predual are exactly those of the form $\mathbb{T}_{n_{1}, m_{1}} \oplus \cdots \oplus \mathbb{T}_{n_{k}, m_{k}}$ (and consequently gives a proof of Theorem 1.1 for the finite dimensional case).

Proposition 5.7. Let $R$ be a von Neumann algebra. Let $X$ be a finited dimensional completely 1-complemented subspace of $R_{*}$. Then $X$ is completely isometric to $\mathbb{T}_{n_{1}, m_{1}} \oplus \cdots \oplus \mathbb{T}_{n_{k}, m_{k}}$ for some integers $n_{i}, m_{i}$.

Proof. If $X$ is completely 1-complemented in $R_{*}$, then $X^{*}$ is completely isometric to a completely 1-complemented subspace of $R$ and thus completely isometric to a TRO, by Youngson's theorem $[\mathbf{Y}]$. But by $[\mathbf{H e}]$, all finite dimensional TROs are completely isometric to spaces of the form $\mathbb{M}_{n_{1}, m_{1}} \oplus \cdots \oplus \mathbb{M}_{n_{k}, m_{k}}$. Hence $X$ is completely isometric to a space of the form $\mathbb{T}_{n_{1}, m_{1}} \oplus \cdots \oplus \mathbb{T}_{n_{k}, m_{k}}$.

\section{Completely contractive projections with finite dimensional range.}

In the context of Proposition 5.6, if $k=1$ and $n_{1}=m_{1}$ (i.e., if $S: \mathbb{T}_{n} \rightarrow R_{*}$ ), then

$$
J_{*}\left(w^{*} \omega\right)=S^{-1}(p(\omega)),
$$

for all $\omega \in R_{*}$, where $J$ and $w$ are as in Proposition 5.4. Hence, $p$ is a generalization of the conditional expectations of the (commutative) $L_{1}$ case. Contractive projections on $L_{1}$ are "perturbations of conditional expectations" (see $[\mathbf{D}]$ ). In this section, we show that this is also true for completely contractive projections on $R_{*}$, with finite dimensional range. The main results are Theorems 6.4 and 6.5.

We first state an elementary lemma whose proof can be found in [Kad] Lemma 1. Recall that for a partial isometry $v \in \mathbb{B}(\mathcal{H}), E(v)$ is the completely contractive projection on $\mathbb{B}(\mathcal{H})$ given by $E(v)(x)={ }_{\mathrm{df}} l(v) x r(v)$ for all $x \in \mathbb{B}(\mathcal{H})$. Here $l(v)$ and $r(v)$ are the left and right support projections of $v$ respectively. 
Lemma 6.1. Let $v \in \mathbb{B}(\mathcal{H})$ be a partial isometry. Then $v$ is an extreme point of the closed unit ball of $E(v) \mathbb{B}(\mathcal{H})$.

Most of the work of this section is in the next lemma, which is a straightforward generalization of a result of Arazy and Friedman (see [AF2]).

Lemma 6.2. Let $R$ be a von Neumann algebra. Let $X \subseteq R_{*}$ be a finite dimensional subspace. Let $p: R_{*} \rightarrow X$ be a contractive projection, onto $X$. Thinking of $p$ as a map from $R_{*}$ to $R_{*}$, let $P^{*}: R \rightarrow R$ be the dual map. Suppose that $\omega_{1}, \omega_{2} \in X$, with

$$
\omega_{1} \perp \omega_{2} .
$$

Then

$$
\left(P^{*}\left(v\left(\omega_{1}\right)^{*}\right)\right)^{*} \perp v\left(\omega_{2}\right) \text { and }\left(P^{*}\left(v\left(\omega_{2}\right)^{*}\right)\right)^{*} \perp v\left(\omega_{1}\right)
$$

where $v\left(\omega_{i}\right)$ is the partial isometry in the polar decomposition of the normal linear functional $\omega_{i}, i=1,2$.

Proof. By symmetry, it suffices to show that $\left(P^{*}\left(v\left(\omega_{1}\right)^{*}\right)\right)^{*} \perp v\left(\omega_{2}\right)$. We have that

$$
P^{*}\left(v\left(\omega_{i}\right)^{*}\right)=v\left(\omega_{i}\right)^{*}+z_{i}^{*},
$$

where $z_{i} \in R,\left\|z_{i}\right\|_{\infty} \leq 1$, and $z_{i} \perp v\left(\omega_{i}\right)$ for $\mathrm{i}=1,2$. Choose a matrix representation (of $\mathbb{B}(\mathcal{H})$ ) in which

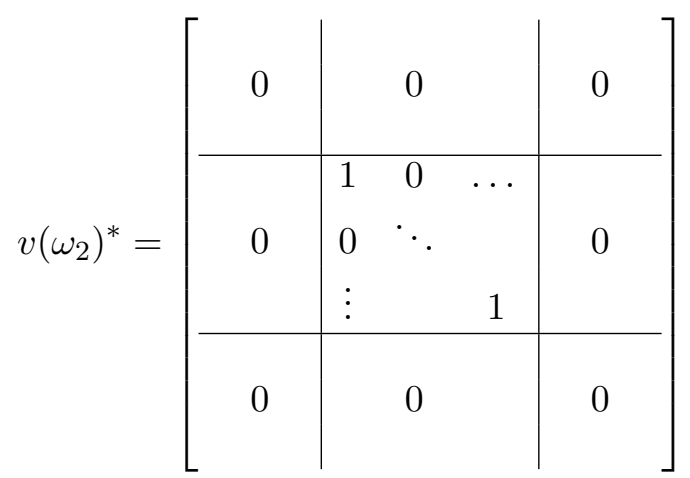

and

$$
P^{*}\left(v\left(\omega_{1}\right)^{*}\right)=\left[\begin{array}{ccc|c|c}
1 & 0 & \ldots & & \\
0 & \ddots & & 0 & 0 \\
\vdots & & 1 & & \\
\hline & 0 & & z_{1,1} & z_{1,2} \\
\hline & 0 & & z_{2,1} & z_{2,2}
\end{array}\right]
$$


where

$$
z_{1}^{*}=\left[\begin{array}{ll}
z_{1,1} & z_{1,2} \\
z_{2,1} & z_{2,2}
\end{array}\right]
$$

Since $P^{*}$ is contractive, we have that

$$
\begin{aligned}
\max \{|s|,|t|\} & =\left\|s v\left(\omega_{1}\right)^{*}+t v\left(\omega_{2}\right)^{*}\right\|_{\infty} \\
& \geq\left\|s P^{*}\left(v\left(\omega_{1}\right)^{*}\right)+t P^{*}\left(v\left(\omega_{2}\right)^{*}\right)\right\|_{\infty},
\end{aligned}
$$

for all $\mathrm{s}, \mathrm{t} \in \mathbf{C}$. Hence for $|s| \leq 1$, we have

$$
\begin{aligned}
1 & \geq\left\|s P^{*}\left(v\left(\omega_{1}\right)^{*}\right)+P^{*}\left(v\left(\omega_{2}\right)^{*}\right)\right\|_{\infty} \\
& =\left\|s v\left(\omega_{1}\right)^{*}+s z_{1}^{*}+v\left(\omega_{2}\right)^{*}+z_{2}^{*}\right\|_{\infty} \\
& \geq\left\|s z_{1,1}+v\left(\omega_{2}\right)^{*}\right\|_{\infty} .
\end{aligned}
$$

So

$$
v\left(\omega_{2}\right)^{*}=(1 / 2)\left(v\left(\omega_{2}\right)^{*}+z_{1,1}\right)+(1 / 2)\left(v\left(\omega_{2}\right)^{*}-z_{1,1}\right),
$$

where

$$
\left\|v\left(\omega_{2}\right)^{*} \pm z_{1,1}\right\|_{\infty} \leq 1 .
$$

But by Lemma $6.1, v\left(\omega_{2}\right)^{*}$ is an extreme point of the closed unit ball of $E\left(v\left(\omega_{2}\right)^{*}\right)(\mathbb{B}(\mathcal{H}))$. Hence, $z_{1,1}=0$. Also, for $|s| \leq 1$,

$$
\begin{aligned}
1 & \geq\left\|\left(s P^{*}\left(v\left(\omega_{1}\right)^{*}\right)+P^{*}\left(v\left(\omega_{2}\right)^{*}\right)\right) l\left(v\left(\omega_{2}\right)\right)\right\|_{\infty}^{2} \\
& =\left\|s P^{*}\left(v\left(\omega_{1}\right)^{*}\right) l\left(v\left(\omega_{2}\right)\right)+P^{*}\left(v\left(\omega_{2}\right)^{*}\right) l\left(v\left(\omega_{2}\right)\right)\right\|_{\infty}^{2} \\
& =\left\|s\left(v\left(\omega_{1}\right)^{*}+z_{1}^{*}\right) l\left(v\left(\omega_{2}\right)\right)+\left(v\left(\omega_{2}\right)^{*}+z_{2}^{*}\right) l\left(v\left(\omega_{2}\right)\right)\right\|_{\infty}^{2} \\
& =\left\|s z_{2,1}+v\left(\omega_{2}\right)^{*}\right\|_{\infty}^{2} .
\end{aligned}
$$

Hence, $z_{2,1}=0$. Similarly, $z_{1,2}=0$. Hence, $z_{1}^{*} \perp v\left(\omega_{2}\right)^{*}$ Hence,

$$
P^{*}\left(v\left(\omega_{1}\right)^{*}\right)=v\left(\omega_{1}\right)^{*}+z_{1}^{*} \perp v\left(\omega_{2}\right)^{*} .
$$

In the remainder of this paper, $v\left(S\left(e_{i, j}^{l}\right)\right)$ will always be the partial isometry in the polar decomposition of the normal linear functional $S\left(e_{i, j}^{l}\right)$.

Recall also the following definitions: Given a von Neumann algebra predual $R_{*}$ and a finite dimensional subspace $X \subseteq R_{*}$,

$$
l(X)=_{\mathrm{df}} \sup \{l(\omega): \omega \in X\},
$$

and

$$
r(X)=_{\mathrm{df}} \sup \{r(\omega): \omega \in X\}
$$


where for $\omega \in R_{*}, l(\omega)$ and $r(\omega)$ are the left and right support projections of $\omega$ respectively. Also, $E(X)$ and $F(X)$ are the completely contractive projections on $R_{*}$ given by

$$
E(X)(\omega)={ }_{\mathrm{df}} l(X) \omega r(X),
$$

and

$$
F(X)=_{\mathrm{df}}(1-l(X)) \omega(1-r(X)),
$$

for all $\omega \in R_{*}$.

Lemma 6.3. Suppose that $p$ is a completely contractive projection on $R_{*}$, with finite dimensional range $X$. Let $P^{*}: R \rightarrow R$ be the dual map. Let $S: \mathbb{T}_{n_{1}, m_{1}} \oplus \cdots \oplus \mathbb{T}_{n_{k}, m_{k}} \rightarrow X$ be a complete isometry onto $X$. For all $i, j$, l, let

$$
P^{*}\left(v\left(S\left(e_{i, j}^{l}\right)\right)^{*}\right)=v\left(S\left(e_{i, j}^{l}\right)\right)^{*}+\left(z_{i, j}^{l}\right)^{*},
$$

where $v\left(S\left(e_{i, j}^{l}\right)\right) \perp z_{i, j}^{l}$ and $\left\|z_{i, j}^{l}\right\| \leq 1$. Then for all $i, j, l, a, b, d$, we have that

$$
v\left(S\left(e_{i, j}^{l}\right)\right) \perp z_{a, b}^{d},
$$

i.e.,

$$
l(X) \perp l\left(z_{a, b}^{d}\right) \text { and } r(X) \perp r\left(z_{a, b}^{d}\right) .
$$

Proof. The case where $n_{l}, m_{l} \geq 2$ follows from Proposition 5.1 and Lemma 6.2. The general case follows in the usual way, by moving up to higher dimensional matrices.

Theorem 6.4. Suppose that we have the same hypothesis as Lemma 6.3. Then

$$
p=q+T
$$

where

$$
q(\omega)={ }_{\mathrm{df}} \sum_{i, j, l}\left\langle\omega \mid v\left(S\left(e_{i, j}^{l}\right)\right)\right\rangle S\left(e_{i, j}^{l}\right)
$$

for all $\omega \in R_{*}$, and

$$
\|T\|_{c b} \leq 1, T^{2}=0 \text { and } T=p T=q T=T F(X) .
$$


Proof.

$$
\begin{aligned}
p(\omega) & =\sum_{i, j, l} \omega\left(P^{*}\left(v\left(S\left(e_{i, j}^{l}\right)\right)^{*}\right)\right) S\left(e_{i, j}^{l}\right) \\
& =\sum_{i, j, l} \omega\left(v\left(S\left(e_{i, j}^{l}\right)\right)^{*}+\left(y_{i, j}^{l}\right)^{*}\right) S\left(e_{i, j}^{l}\right) \\
& =\sum_{i, j, l}\left\langle\omega \mid v\left(S\left(e_{i, j}^{l}\right)\right)\right\rangle S\left(e_{i, j}^{l}\right)+\sum_{i, j, l}\left\langle\omega \mid y_{i, j}^{l}\right\rangle S\left(e_{i, j}^{l}\right) \\
& =q(\omega)+T(\omega),
\end{aligned}
$$

where

$$
T(\omega)=p F(X) \omega=\sum_{i, j, l}\left\langle\omega \mid y_{i, j}^{l}\right\rangle S\left(e_{i, j}^{l}\right),
$$

by Lemma 6.3. Also, note that $q=p E(X)$, by Lemma 6.3. It is now not hard to check that $T$ satisfies the required properties.

Theorem 6.5. Suppose that $X \subseteq R_{*}$ is a finite dimensional subspace such that there is a complete isometry $S: \mathbb{T}_{n_{1}, m_{1}} \oplus \cdots \oplus \mathbb{T}_{n_{k}, m_{k}} \rightarrow X$ onto $X$. Let $q: R_{*} \rightarrow X$ be the completely contractive projection give by

$$
q(\omega)=_{\mathrm{df}} \sum_{i, j, l}\left\langle\omega \mid v\left(S\left(e_{i, j}^{l}\right)\right)\right\rangle S\left(e_{i, j}^{l}\right),
$$

for all $\omega \in R_{*}$. Let $T$ be an operator on $R_{*}$ such that

$$
\|T\|_{c b} \leq 1, T^{2}=0 \text { and } T=p T=q T=T F(X) .
$$

Then

$$
p=q+T: R_{*} \rightarrow X
$$

is a completely contractive projection onto $X$.

Proof. Firstly, it is not hard to check that $p$ is a projection. Since $p=$ $q+T=q+q T$, we have that

$$
\operatorname{ran}(p) \subseteq \operatorname{ran}(q)
$$

But since $p T=(q+T) T=q T+T^{2}=q T=T$, we have that $q=p-T=$ $p-p T$; and hence

$$
\operatorname{ran}(q) \subseteq \operatorname{ran}(p)
$$

Thus,

$$
\operatorname{ran}(p)=\operatorname{ran}(q)
$$


Also, for $\omega \in R_{*}$,

$$
\begin{aligned}
\|p(\omega)\|_{1} & =\|(q+T) \omega\|_{1} \\
& \leq\|q \omega\|_{1}+\|T \omega\|_{1} \\
& =\|q E(X) \omega\|_{1}+\|T F(X) \omega\|_{1} \\
& \leq\|E(X) \omega\|_{1}+\|F(X) \omega\|_{1} \quad \\
& =\|(E(X)+F(X)) \omega\|_{1} \quad \text { by Proposition } 5.3 \\
& \leq\|\omega\|_{1} .
\end{aligned}
$$

Hence, $p$ is contractive. A similar argument shows that $p$ is completely contractive, and we are done.

\section{References}

[AF1] J. Arazy and Y. Friedman, The isometries of $C_{p}^{n, m}$ into $C_{p}$, Israel J. Math., 26 (1977), 151-165, MR 55 \#13271, Zbl 0345.47035.

[AF2] Contractive projections in $C_{1}$ and $C_{\infty}$, Memoirs AMS, 13(200) (1978), MR 82b:47023, Zbl 0382.47020.

[AF3] _ Contractive projections in $C_{p}$, Memoirs AMS, 95(459) (1992), MR 92f:47033, Zbl 0748.47040.

[Arv] W. Arveson, Subalgebras of $C^{*}$-algebras, Acta Math., 123 (1969), 141-224, MR 40 \#6274, Zbl 0194.15701.

[C] M.-D. Choi, A Schwarz inequality for positive linear maps on $C^{*}$-algebras, Illinois J. Math., 18 (1974), 565-574, MR 50 \#8089, Zbl 0293.46043.

[D] R.G. Douglas, Contractive projections on an $L_{1}$-space, Pacific J. Math., 15 (1965), 443-462, MR 32 \#4541, Zbl 0148.12203.

[EOR] E. Effros, N. Ozawa and Z.-J. Ruan, On injectivity and nuclearity for operator spaces, Duke Math. J., 110(3) (2001), 489-521, CMP 1869114.

[ER1] E. Effros and Z.-J. Ruan, On approximation properties for operator spaces, Internat. J. Math., 1(2) (1990), 163-187, MR 92g:46089, Zbl 0747.46014.

[ER2] $\quad \mathcal{O L}_{p}$ spaces, Contemporary Mathematics, American Math. Soc., 228 (1998), 51-77, MR 2000a:46102, Zbl 0929.46048.

[FR] Y. Friedman and B. Russo, Solution of the contractive projection problem, J. Funct. Anal., 60(1) (1985), 56-79, MR 87a:46115, Zbl 0558.46035.

[H] M. Hamana, Injective envelopes of dynamical systems, preprint, April 1991.

[Harr] L.A. Harris, Bounded symmetric homogeneous domains in infinite dimensional spaces, Proceedings on Infinite Dimensional Holomorphy (Internat. Conf., Univ. Kentucky, Lexington, Ky., 1973), 13-40; Lecture Notes in Math., 364, Springer, Berlin, 1974, MR 53 \#11106, Zbl 0293.46049.

[He] M.R. Hestenes, On a Ternary Algebra, Collection of articles dedicated to the memory of Abraham Adrian Albert, Scripta Math., 29(3-4) (1973), 253-272, MR 56 \#3174, Zbl 0275.20120.

[Kad] R.V. Kadison, Isometries of operator algebras, Ann. of Math., 54 (1951), 325-338, MR 13,256a, Zbl 0045.06201. 
[K1] E. Kirchberg, On restricted perturbations in inverse images and a description of normalizer algebras in $C^{*}$-algebras, J. Funct. Anal., 129(1) (1995), 1-34, MR 95m:46094a, Zbl 0828.46052.

[K2] - On non-semisplit extensions, tensor products and exactness of group $C^{*}$ algebras, Invent. Math., 112 (1993), 449-489, MR 94d:46058, Zbl 0803.46071.

[P] V. Paulsen, Completely bounded maps and dilations, Pitman Research Notes in Math., 146, Longman, Wiley, New York, 1986, MR 88h:46111, Zbl 0614.47006.

[R] Z.-J. Ruan, Subspaces of $C^{*}$-algebras, J. Funct. Anal., 76 (1988), 217-230, MR 89h:46082, Zbl 0646.46055.

[Tak] M. Takesaki, Theory of Operator Algebras I, Springer, Berlin, Heidelberg, and New York, 1979, MR 81e:46038, Zbl 0436.46043.

[Yead] F. Yeadon, Isometries of non-commutative $L_{p}$-spaces, Math. Proc. Cambridge Phil. Soc., 90 (1981), 41-50, MR 82g:46108, Zbl 0483.46041.

[Y] M.A. Youngson, Completely contractive projections on $C^{*}$-algebras, Quart. J. Math. Oxford Ser. (2), 34 (1983), 507-511, MR 85f:46112, Zbl 0542.46029.

Received February 14, 2000 and revised October 16, 2001. Part of this research was carried out while the authors were visiting L'Institut Henri Poincaré (IHP). The authors acknowledge the kind hospitality of the IHP. Part of this work is from the thesis of the first author. The first author thanks his advisor Dr. Edward Effros for bringing the theorem of Douglas to his attention. The second author is supported by the Japanese Society for the Promotion of Science. The authors also thank the referee for many helpful comments. This work was completed while the first author was a graduate student of the University of California, Los Angeles.

Department of Mathematics

UNIVERSITY OF TORONTO

TOROnTO, OnTARio

CANADA M5S 3G3

E-mail address: png@math.toronto.edu

Department of Mathematical Science

UNIVERSITY OF TOKYO

KOMABA 153-8914, JAPAN

E-mail address: narutaka@ms.u-tokyo.ac.jp 